\title{
EVALUATION OF BIO-EFFICIENCY AND PHYTOTOXICITY OF PENDIMETHALIN 38.7\% CS (STOMP) IN COTTON
}

\author{
RAMAN. R and R. KRISHNAMOORTHY \\ Department of Agronomy, Faculty of Agriculture, Annamalai University, \\ Annamalai nagar, Chidambaram - 608002, Tamil Nadu, India. \\ DOI: https://doi.org/10.51193/IJAER.2021.7104
}

\begin{abstract}
Field experiments were conducted at the Experimental Farm, Department of Agronomy, Annamalai University, Annamalai nagar to study the evaluation of Pendimethalin $38.7 \%$ CS and various cotton herbicides in cotton during August 2018 to January 2019 and August 2019 to January 2020 with cv. MCU 7. The experiment consists of eight treatment viz., Pendimethalin $38.7 \%$ CS @ 438.75 g a.i/ha, Pendimethalin 38.7\% CS @ $580.50 \mathrm{~g}$ a.i/ha, Pendimethalin 38.7 \% CS@677.25 g a.i/ha, Fluchloralin 45\% EC @ $1125 \mathrm{~g}$ a.i/ha, Pendimethalin 30 \% EC @ 750 g a.i/ha, Trifluralin 48\% EC @ 960 g a.i/ha, Fluchloralin 45\% EC @ $2000 \mathrm{~g}$ a.i/ha and unweeded check. Regardless of season, all the treatments were found to significantly influenced the weed biometrics, growth, yield components and yield of cotton. Among the treatments tested, Pendimethalin 38.7\% CS @ 677.25 g a.i/ha recorded least weed counts, weed dry matter production, highest weed control index and weed control efficiency favouring higher growth, yield attributes and seed cotton yield in first and second seasons, respectively. This was followed by Pendimethalin $38.7 \%$ CS @ $580.50 \mathrm{~g}$ a.i/ha. These treatments were significantly superior than the rest in reducing the weed infestation and ultimately increasing seed cotton yield in both seasons. Unweeded check recorded the highest weed counts, weed biomass resulting in the least growth and seed cotton yield in first and second seasons, respectively. From the study, it may be concluded that application of Pendimethalin 38.7\% CS @ 677.25 g a.i/ha is considered to be judicious recommendation to cotton farmers in view of inadequate labour and higher manual weeding cost.
\end{abstract}

Keywords: Cotton, Herbicides, Phytotoxicity, Yield. 
International Journal of Agriculture and Environmental Research

ISSN: 2455-6939

Volume: 07, Issue: 01 "January-February 2021"

\section{INTRODUCTION}

Cotton the "white gold" is the one among the most important commercial fiber crop as well as the cash crop of India. It contributes about $85 \%$ of raw materials for manufacture of textiles in the world (Dilbaugh Muhammad et al., 2009). Globally it is cultivated over an area of 34.6 million hectare with the production of 25.1 million tonnes in world 2008-2009 (Cotton Corporation of India, 2009). India is the second largest cotton growing country in the world next to China, produces around $16 \%$ of the world's total production. The area under cotton is 93.73 lakh hectares, production of 290 lakh bales with the productivity of $526 \mathrm{~kg} / \mathrm{ha}$. In Tamil Nadu, it is grown in an area of 1.20 lakh hectares with a production of 5 lakh bales and the productivity of $708 \mathrm{~kg} / \mathrm{ha}$. Weeds cause enormous losses and suffering to human beings by way of reduction in crop yield and quality, wastage of human energy and resources and increased cost of cultivation (Gurbachan Singh, 2005). Pendimethalin, Trifluralin and Fluchloralin is also gaining momentum among cotton growers. Among these herbicides, Pendimethalin 33\% is a direct dinitroaline herbicides commonly used to control grasses and small seeded dicot weed species as preemergence (PRE) or pre-plant incorporation (PPI). Gill et al., (1996) noted that when Pendimethalin applied on dry land and irrigated crop immediately, exhibited $81 \%$ weed control and increased seed cotton yield as compared to delayed irrigation. This study was undertaken to evaluate Pendimethalin $38.7 \%$ CS to control weeds in cotton and to find out its phytotoxic effect in cotton crop

\section{MATERIALS AND METHODS}

The experiment was conducted at the Agriculture Experimental farm, Department of Agronomy, Annamalai University, Tamil Nadu during August 2008 to January 2009 and August 2009 to January 2010. The Experimental farm is situated at $11^{\circ} 24^{\prime} \mathrm{N}$ latitude, $79^{\circ} 44^{\prime} \mathrm{E}$ longitude and at an altitude of +5.79 meters above mean sea level. The soil of the experimental site was clay loam in texture with $258.5 \mathrm{~kg} / \mathrm{ha}$ available $\mathrm{N}, 17.3 \mathrm{~kg} / \mathrm{ha} \mathrm{P}$ and $310.0 \mathrm{~kg} / \mathrm{ha} \mathrm{K}$ and $7.4 \mathrm{pH}$ and 1.20 $\mathrm{dS} / \mathrm{m}$ EC at the start of the experiment. The experiment was laid out in randomized block design with 3 replications. There were 7 weed control treatments, viz. Pendimethalin $38.7 \%$ CS @ $438.75 \mathrm{~g}$ a.i/ha, Pendimethalin 38.7 \% CS @ $580.50 \mathrm{~g}$ a.i/ha, Pendimethalin $38.7 \%$ CS @ $677.25 \mathrm{~g}$ a.i/ha, Fluchloralin 45\% EC @ 1125 g a.i/ha, Pendimethalin 30 \% EC @ $750 \mathrm{~g}$ a.i/ha, Trifluralin 48\% EC @ 960 g a.i/ha, Fluchloralin 45\% EC @ 2000 g a.i/ha and unweeded check. The seeds of cotton were dibbled with a spacing of $60 \mathrm{~cm}$ between rows and $30 \mathrm{~cm}$ between plants at a depth of 2 to $3 \mathrm{~cm}$ and then covered with the soil. The herbicides Pendimethalin, Fluchloralin and Trifluralin applied @ 1.5 lit a.i/ha were mixed with water @ 500 lit required doses. These pre-emergence herbicides were sprayed three days after sowing of the cotton seeds by a knapsack sprayer uniformly over the soil. The crop was raised by using recommended 
package of practices. The seed cotton was harvested from the well opened bolls from 110 DAS onwards and seven pickings were given for the first crop, whereas only four pickings were given for second crop at weekly intervals. The harvested seed cotton was shade dried on a layer of river sand and weighed at uniform moisture content. Economics of cotton was calculated by using prevailing market prices of inputs and outputs under different weed-control treatments.

\section{RESULTS AND DISCUSSION}

\section{Weed count:}

The major weed flora observed in cotton field consisted of Cyperus rotundus, Trianthema portulacastrum, Phyllanthus maderaspatensis, Corchorus trilocularis, Convolvulus arvensis, Eclipta alba, Cleome viscose, Tridax procumbens, Echinochloa colonum and Cynodan dactylon. All the treatments were effective in controlling the weeds and caused significant reduction in their population. Among the various herbicides, Pendimethalin $38.7 \%$ CS @ $677.25 \mathrm{~g}$ a.i/ha was very effective and significantly superior to the rest of the treatments in respect of weed count in both seasons. Effective weed control measures such as pre emergence application of Pendimethalin at 3 DAS might have helped in minimizing weed population and by reducing weed competition for light, moisture and nutrients as compared to all other herbicide treatments. Weed density were reduced further due to increase in the rates of Pendimethalin application. Pendimethalin, irrespective of rates of application, provided better weed control than other herbicides. Pendimethalin 30 EC also reduced the weed count and this result was in consonance with the findings of Naqib Ullabkhan et al., (2001). The highest weed population was recorded under the unweeded check treatment.

Table 1: Effect of weed-control treatments on weed count $\left(\mathrm{Nos} . / \mathrm{m}^{2}\right)$ in cotton.

\begin{tabular}{|c|c|c|c|c|c|c|}
\hline \multirow[b]{2}{*}{ Treatment } & \multicolumn{2}{|c|}{30 DAS } & \multicolumn{2}{|c|}{60 DAS } & \multicolumn{2}{|c|}{90 DAS } \\
\hline & $\begin{array}{l}\text { First } \\
\text { year }\end{array}$ & $\begin{array}{c}\text { Second } \\
\text { year }\end{array}$ & $\begin{array}{l}\text { First } \\
\text { year }\end{array}$ & Second year & First year & Second year \\
\hline $\begin{array}{l}\text { Pendimethalin } \\
38.7 \% \text { CS @ } \\
438.75 \mathrm{~g} \\
\text { a.i/ha }\end{array}$ & $\begin{array}{l}20.57 \\
(4.59)\end{array}$ & $26.22(5.17)$ & $\begin{array}{l}24.53 \\
(5.01)\end{array}$ & $31.64(5.66)$ & $\begin{array}{l}29.80 \\
(5.17)\end{array}$ & $42.42(6.53)$ \\
\hline $\begin{array}{l}\text { Pendimethalin } \\
38.7 \% \mathrm{CS} @ \\
580.50 \mathrm{~g} \\
\text { a.i/ha }\end{array}$ & $\begin{array}{l}13.25 \\
(3.70)\end{array}$ & $18.81(4.39)$ & $\begin{array}{l}16.71 \\
(4.14)\end{array}$ & $22.88(4.77)$ & $\begin{array}{l}21.07 \\
(4.64)\end{array}$ & $31.08(5.61)$ \\
\hline Pendimethalin & 10.13 & $15.65(4.02)$ & 13.49 & $18.69(4.38)$ & 17.09 & $25.91(5.13)$ \\
\hline
\end{tabular}


International Journal of Agriculture and Environmental Research

ISSN: 2455-6939

Volume: 07, Issue: 01 "January-February 2021"

\begin{tabular}{|c|c|c|c|c|c|c|}
\hline $\begin{array}{l}38.7 \% \text { CS @ } \\
677.25 \mathrm{~g} \\
\text { a.i/ha }\end{array}$ & (3.26) & & (3.74) & & $(4.20)$ & \\
\hline $\begin{array}{l}\text { Fluchloralin } \\
45 \% \text { EC @ } \\
1125 \mathrm{~g} \mathrm{a.i/ha}\end{array}$ & $\begin{array}{l}27.85 \\
(5.32)\end{array}$ & $33.22(5.80)$ & $\begin{array}{l}31.65 \\
(5.67)\end{array}$ & $41.67(6.49)$ & $\begin{array}{l}40.44 \\
(6.39)\end{array}$ & $56.37(7.54)$ \\
\hline $\begin{array}{l}\text { Pendimethalin } \\
30 \% \text { EC @ } \\
750 \text { g a.i/ha }\end{array}$ & $\begin{array}{l}17.35 \\
(4.22)\end{array}$ & $22.99(4.84)$ & $\begin{array}{l}21.02 \\
(4.63)\end{array}$ & $26.92(5.24)$ & $\begin{array}{l}25.70 \\
(5.11)\end{array}$ & $36.22(6.05)$ \\
\hline $\begin{array}{l}\text { Trifluralin } \\
\text { 48\% EC @ } \\
960 \text { g a.i/ha }\end{array}$ & $\begin{array}{l}31.95 \\
(5.10)\end{array}$ & $37.31(6.14)$ & $\begin{array}{l}35.98 \\
(6.03)\end{array}$ & $46.69(6.86)$ & $\begin{array}{l}47.54 \\
(6.93)\end{array}$ & $63.42(7.99)$ \\
\hline $\begin{array}{l}\text { Fluchloralin } \\
\text { 45\% EC @ } \\
2000 \text { g a.i/ha }\end{array}$ & $\begin{array}{l}24.13 \\
(4.96)\end{array}$ & 29.67 (5.49) & $\begin{array}{l}28.15 \\
(5.35)\end{array}$ & $36.52(6.08)$ & $\begin{array}{l}35.03 \\
(5.93)\end{array}$ & $49.55(7.08)$ \\
\hline $\begin{array}{l}\text { Unweeded } \\
\text { check }\end{array}$ & $\begin{array}{l}35.83 \\
(6.02)\end{array}$ & $40.97(6.43)$ & $\begin{array}{l}40.15 \\
(6.37)\end{array}$ & $51.58(7.21)$ & $\begin{array}{l}55.97 \\
(7.49)\end{array}$ & $71.19(8.46)$ \\
\hline SEm \pm & 0.10 & 0.12 & 0.22 & 0.25 & 0.35 & 0.37 \\
\hline $\mathrm{CD}(\mathrm{P}=0.05)$ & 0.21 & 0.25 & 0.45 & 0.51 & 0.71 & 0.75 \\
\hline
\end{tabular}

\section{Weed dry matter:}

The weed control treatments significantly influenced the total weed dry matter during both the years. Pendimethalin 38.7\% CS @ $677.25 \mathrm{~g}$ a.i/ha recorded the lowest weed DMP in both the seasons. This treatment was superior to the other herbicides tested as it controlled grasses, sedges and broad leaved weeds in the critical stages. This might be due to restriction of emergence of fresh weeds in the later stages. Effective control of weeds, due to blocking of their perennation process and inhibition of their germination and growth (Hadizades et al., 2002) might have resulted in lesser weed DMP. Lesser weed DMP in this treatment were due to better control of weeds especially in the early stages of crop growth. Unweeded check recorded the highest weeds DMP than other weed control treatment. This findings are in a great agreement with the work of Panwar et al., (2001) who reported the efficacy of Pendimethalin for controlling weeds in cotton. The findings of Hiremath and Rao (2001) further support the present findings. Richardson et al., (2007) who reported that the weeds are controlled more efficiently by the application of Pendimethalin. 
International Journal of Agriculture and Environmental Research

ISSN: 2455-6939

Volume: 07, Issue: 01 "January-February 2021"

Table 2: Effect of weed-control treatments on weed dry matter production $(\mathrm{kg} / \mathrm{ha})$ in cotton.

\begin{tabular}{|c|c|c|c|c|c|c|}
\hline \multirow[b]{2}{*}{ Treatment } & \multicolumn{2}{|c|}{ 30 DAS } & \multicolumn{2}{|c|}{ 60 DAS } & \multicolumn{2}{|c|}{90 DAS } \\
\hline & First year & $\begin{array}{c}\text { Second } \\
\text { year }\end{array}$ & $\begin{array}{l}\text { First } \\
\text { year }\end{array}$ & Second year & First year & Second year \\
\hline $\begin{array}{l}\text { Pendimethalin } \\
38.7 \% \text { CS @ } \\
438.75 \mathrm{~g} \\
\text { a.i/ha }\end{array}$ & 140.30 & 155.93 & 179.88 & 209.22 & 261.96 & 333.23 \\
\hline $\begin{array}{l}\text { Pendimethalin } \\
38.7 \% \text { CS @ } \\
580.50 \mathrm{~g} \\
\text { a.i/ha }\end{array}$ & 90.56 & 101.19 & 118.42 & 137.44 & 166.54 & 200.90 \\
\hline $\begin{array}{l}\text { Pendimethalin } \\
38.7 \% \text { CS @ } \\
677.25 \mathrm{~g} \\
\text { a.i/ha }\end{array}$ & 56.48 & 61.03 & 73.12 & 86.03 & 92.09 & 115.27 \\
\hline $\begin{array}{l}\text { Fluchloralin } \\
45 \% \text { EC @ } \\
1125 \text { g a.i/ha }\end{array}$ & 187.20 & 212.57 & 244.65 & 284.86 & 359.59 & 494.88 \\
\hline $\begin{array}{l}\text { Pendimethalin } \\
30 \% \text { EC @ } \\
750 \text { g a.i/ha }\end{array}$ & 125.18 & 140.31 & 163.67 & 189.06 & 241.65 & 287.58 \\
\hline $\begin{array}{l}\text { Trifluralin } \\
48 \% \text { EC @ } \\
960 \text { g a.i/ha }\end{array}$ & 217.36 & 253.52 & 290.63 & 338.54 & 434.61 & 515.55 \\
\hline $\begin{array}{l}\text { Fluchloralin } \\
45 \% \text { EC @ } \\
2000 \text { g a.i/ha }\end{array}$ & 172.95 & 196.18 & 225.53 & 261.25 & 332.61 & 414.01 \\
\hline $\begin{array}{l}\text { Unweeded } \\
\text { check }\end{array}$ & 285.49 & 325.68 & 357.50 & 419.55 & 534.87 & 604.52 \\
\hline SEm \pm & 16.03 & 19.01 & 22.60 & 25.70 & 37.55 & 44.30 \\
\hline $\mathrm{CD}(\mathrm{P}=0.05)$ & 32.06 & 38.03 & 45.27 & 51.41 & 75.11 & 88.63 \\
\hline
\end{tabular}




\section{Seed cotton yield:}

All the treatments had a significant impact on the yield attributes of cotton. Among the herbicides tested, Pendimethalin 38.7\% CS @ 677.25 g a.i/ha registered maximum yield and yield components than other herbicide treatments. It could be attributed to significantly lower weed population, dry matter accumulation of weeds and hence higher number of sympoidal branches, number of monopoidal branches and number of bolls per plant in this treatment. The superior performance of Pendimethalin might be attributed to reduce crop-weed competition in the initial stages which helped in synchronization of number of sympoidal branches, number of monopoidal branches and number of bolls per plant. The lowest boll weight was recorded in weedy check by weak plants suppressed by high weed infestation. These are in line with the findings of Gill et al., (1996), Hassan et al., (1996) and Khan et al., (2001). The increase in yield might be due to effective control of weeds which reduced the crop-weed competition by effective weed control measures such as pre-emergence application of Pendimethalin at 3 DAS offering efficient and prolonged weed control might have favoured the crop with better rooting, higher LAI, pre and post flowering photosynthesis and yield attributes. Severe competition of weeds in unweeded check results in suppression of crop growth and there by reduction in seed cotton yield in both seasons.

Table 3: Effect of weed-control treatments on yield and yield attributes of cotton.

\begin{tabular}{|c|c|c|c|c|c|c|c|c|c|c|}
\hline \multirow{2}{*}{ Treatment } & \multicolumn{2}{|c|}{$\begin{array}{c}\text { No. of } \\
\text { monopodial } \\
\text { branches/plant }\end{array}$} & \multicolumn{2}{|c|}{$\begin{array}{c}\text { No. of } \\
\text { sympodial } \\
\text { branches/plan } \\
\mathbf{t}\end{array}$} & \multicolumn{2}{|c|}{$\begin{array}{c}\text { No. of } \\
\text { bolls/plant }\end{array}$} & \multicolumn{2}{|c|}{$\begin{array}{l}\text { Boll weight } \\
\text { (g) }\end{array}$} & \multicolumn{2}{|c|}{$\begin{array}{l}\text { Seed cotton } \\
\text { yield }(\mathrm{kg} / \mathrm{ha})\end{array}$} \\
\hline & $\begin{array}{l}\text { First } \\
\text { year }\end{array}$ & $\begin{array}{l}\text { Secon } \\
\text { d year }\end{array}$ & $\begin{array}{l}\text { First } \\
\text { year }\end{array}$ & $\begin{array}{l}\text { Secon } \\
\text { d year }\end{array}$ & $\begin{array}{l}\text { First } \\
\text { year }\end{array}$ & $\begin{array}{c}\text { Seco } \\
\text { nd } \\
\text { year }\end{array}$ & $\begin{array}{l}\text { First } \\
\text { year }\end{array}$ & $\begin{array}{c}\text { Seco } \\
\text { nd } \\
\text { year }\end{array}$ & $\begin{array}{l}\text { First } \\
\text { year }\end{array}$ & $\begin{array}{c}\text { Seco } \\
\text { nd } \\
\text { year }\end{array}$ \\
\hline $\begin{array}{l}\text { Pendimethalin } \\
38.7 \% \text { CS @ } \\
438.75 \mathrm{~g} \\
\text { a.i/ha }\end{array}$ & 4.12 & 3.59 & 19.73 & 18.29 & 26.86 & 25.57 & 3.59 & 3.53 & 1778 & 1706 \\
\hline $\begin{array}{l}\text { Pendimethalin } \\
38.7 \% \text { CS @ } \\
580.50 \mathrm{~g} \\
\text { a.i/ha }\end{array}$ & 5.0 & 4.29 & 22.17 & 21.36 & 30.85 & 29.47 & 3.84 & 3.69 & 2032 & 1933 \\
\hline $\begin{array}{l}\text { Pendimethalin } \\
38.7 \% \text { CS @ }\end{array}$ & 5.42 & 4.62 & 23.26 & 22.46 & 32.84 & 31.29 & 3.97 & 3.78 & 2160 & 2035 \\
\hline
\end{tabular}


International Journal of Agriculture and Environmental Research

ISSN: 2455-6939

Volume: 07, Issue: 01 "January-February 2021"

\begin{tabular}{|l|c|c|c|c|c|c|c|c|c|c|}
\hline $\begin{array}{l}\text { 677.25 g } \\
\text { a.i/ha }\end{array}$ & & & & & & & & & \\
\hline $\begin{array}{l}\text { Fluchloralin } \\
45 \% \text { EC @ } \\
1125 \text { g a.i/ha }\end{array}$ & 3.25 & 2.92 & 16.68 & 15.49 & 23.21 & 21.85 & 3.33 & 3.25 & 1538 & 1473 \\
\hline $\begin{array}{l}\text { Pendimethalin } \\
30 \% \text { EC @ } \\
750 \text { g a.i/ha }\end{array}$ & 4.58 & 3.95 & 21.05 & 19.21 & 28.75 & 27.62 & 3.68 & 3.63 & 1897 & 1821 \\
\hline $\begin{array}{l}\text { Trifluralin } \\
48 \% \text { EC @ } \\
960 \text { g a.i/ha }\end{array}$ & 2.83 & 2.56 & 15.33 & 14.34 & 21.76 & 20.40 & 3.24 & 3.16 & 1433 & 1356 \\
\hline $\begin{array}{l}\text { Fluchloralin } \\
45 \% \text { EC @ } \\
\text { 2000 g a.i/ha }\end{array}$ & 3.68 & 3.25 & 18.27 & 16.94 & 24.89 & 23.95 & 3.43 & 3.33 & 1660 & 1590 \\
\hline $\begin{array}{l}\text { Unweeded } \\
\text { check }\end{array}$ & 2.38 & 2.21 & 14.13 & 13.13 & 19.66 & 18.75 & 3.09 & 3.03 & 1318 & 1236 \\
\hline SEm \pm & 0.19 & 0.15 & 0.49 & 0.42 & 0.57 & 0.54 & 0.035 & 0.025 & 50.39 & 49 \\
\hline CD (P=0.05) & 0.41 & 0.32 & 0.99 & 0.87 & 1.14 & 1.08 & 0.07 & 0.05 & 101 & 98 \\
\hline
\end{tabular}

\section{Economics:}

Among the weed control treatments, the highest gross income was registered with Pendimethalin $38.7 \%$ CS @ 677.25 g a.i/ha in both the seasons compared to other treatments. Increased seed cotton yield due to application of Pendimethalin, efficient weed control measures in right time with the right method might have been responsible for increased return per rupee invested in the best treatment. All weed control treatments had higher gross and net returns than the weedy check during both the years. The benefit: cost ratio under different treatments varied from $1.95-$ 2.96 as compared to 1.83 under weedy check. It may be concluded from this study that weeds from cotton can be controlled effectively by using Pendimethalin 38.7 \% CS @ 677.25 g a.i/ha. 
International Journal of Agriculture and Environmental Research

ISSN: 2455-6939

Volume: 07, Issue: 01 "January-February 2021"

Table 4: Effect of weed-control treatments on economics of cotton.

\begin{tabular}{|c|c|c|c|c|c|c|c|c|}
\hline \multirow{2}{*}{ Treatment } & \multicolumn{2}{|c|}{$\begin{array}{c}\text { Cost of } \\
\text { cultivation } \\
(\mathbf{R s} / \mathbf{h a})\end{array}$} & \multicolumn{2}{|c|}{$\begin{array}{c}\text { Gross income } \\
\text { (Rs/ha) }\end{array}$} & \multicolumn{2}{|c|}{$\begin{array}{l}\text { Net income } \\
\text { (Rs/ha) }\end{array}$} & \multicolumn{2}{|c|}{$\begin{array}{l}\text { Benefit: cost } \\
\text { ratio }\end{array}$} \\
\hline & $\begin{array}{l}\text { First } \\
\text { year }\end{array}$ & $\begin{array}{l}\text { Secon } \\
\text { d year }\end{array}$ & $\begin{array}{l}\text { First } \\
\text { year }\end{array}$ & $\begin{array}{l}\text { Secon } \\
\text { d year }\end{array}$ & $\begin{array}{l}\text { First } \\
\text { year }\end{array}$ & $\begin{array}{c}\text { Seco } \\
\text { nd } \\
\text { year }\end{array}$ & $\begin{array}{l}\text { First } \\
\text { year }\end{array}$ & $\begin{array}{c}\text { Seco } \\
\text { nd } \\
\text { year }\end{array}$ \\
\hline $\begin{array}{l}\text { Pendimethalin } \\
38.7 \% \text { CS @ } \\
438.75 \mathrm{~g} \\
\text { a.i/ha }\end{array}$ & $\begin{array}{c}20307 . \\
74\end{array}$ & $\begin{array}{c}20307 . \\
74\end{array}$ & $\begin{array}{c}4978 \\
4\end{array}$ & 47768 & $\begin{array}{l}2947 \\
6.26\end{array}$ & $\begin{array}{l}2746 \\
0.26\end{array}$ & 2.45 & 2.35 \\
\hline $\begin{array}{l}\text { Pendimethalin } \\
38.7 \% \text { CS @ } \\
580.50 \mathrm{~g} \\
\text { a.i/ha }\end{array}$ & $\begin{array}{c}20371 . \\
53\end{array}$ & $\begin{array}{c}20371 . \\
53\end{array}$ & $\begin{array}{c}5689 \\
6\end{array}$ & 54124 & $\begin{array}{l}3652 \\
4.47\end{array}$ & $\begin{array}{l}3375 \\
2.47\end{array}$ & 2.79 & 2.65 \\
\hline $\begin{array}{l}\text { Pendimethalin } \\
38.7 \% \text { CS @ } \\
677.25 \mathrm{~g} \\
\text { a.i/ha }\end{array}$ & $\begin{array}{c}20415 . \\
07\end{array}$ & $\begin{array}{c}20415 . \\
07\end{array}$ & $\begin{array}{c}6048 \\
0\end{array}$ & 56980 & $\begin{array}{l}4006 \\
4.93\end{array}$ & $\begin{array}{l}3656 \\
4.93\end{array}$ & 2.96 & 2.79 \\
\hline $\begin{array}{l}\text { Fluchloralin } \\
45 \% \text { EC @ } \\
1125 \text { g a.i/ha }\end{array}$ & $\begin{array}{c}20751 . \\
56\end{array}$ & $\begin{array}{c}20751 . \\
56\end{array}$ & $\begin{array}{c}4306 \\
4\end{array}$ & 41244 & $\begin{array}{l}2231 \\
2.44\end{array}$ & $\begin{array}{l}2049 \\
2.44\end{array}$ & 2.07 & 1.98 \\
\hline $\begin{array}{l}\text { Pendimethalin } \\
\text { 30\% EC @ } \\
750 \text { g a.i/ha }\end{array}$ & $\begin{array}{c}20455 . \\
31\end{array}$ & $\begin{array}{c}20455 . \\
31\end{array}$ & $\begin{array}{c}5311 \\
6\end{array}$ & 50988 & $\begin{array}{l}3266 \\
0.69\end{array}$ & $\begin{array}{l}3053 \\
2.69\end{array}$ & 2.59 & 2.49 \\
\hline $\begin{array}{l}\text { Trifluralin } \\
48 \% \text { EC @ } \\
960 \text { g a.i/ha }\end{array}$ & $\begin{array}{c}20570 . \\
39\end{array}$ & $\begin{array}{c}20570 . \\
39\end{array}$ & $\begin{array}{c}4040 \\
4\end{array}$ & 17968 & $\begin{array}{l}1955 \\
3.61\end{array}$ & $\begin{array}{l}1739 \\
7.61\end{array}$ & 1.95 & 1.84 \\
\hline $\begin{array}{l}\text { Fluchloralin } \\
45 \% \text { EC @ } \\
2000 \text { g a.i/ha }\end{array}$ & $\begin{array}{c}21250 . \\
31\end{array}$ & $\begin{array}{c}21250 . \\
31\end{array}$ & $\begin{array}{c}4648 \\
0\end{array}$ & 44520 & $\begin{array}{l}2522 \\
9.69\end{array}$ & $\begin{array}{l}2326 \\
9.69\end{array}$ & 2.18 & 2.09 \\
\hline $\begin{array}{l}\text { Unweeded } \\
\text { check }\end{array}$ & $\begin{array}{c}20110 . \\
31\end{array}$ & $\begin{array}{c}20110 . \\
31\end{array}$ & $\begin{array}{c}3690 \\
4\end{array}$ & 34608 & $\begin{array}{l}1679 \\
3.69\end{array}$ & $\begin{array}{l}1449 \\
7.69\end{array}$ & 1.83 & 1.72 \\
\hline
\end{tabular}


International Journal of Agriculture and Environmental Research

ISSN: 2455-6939

Volume: 07, Issue: 01 "January-February 2021"

\section{REFERENCES}

Dilbaugh Muhammad, Muhammad Naveed Afzal, Ilyas Raza and Muhammad Azam Mian. 2009. Growth and development of cotton (Gossypium hirsutum L.) as affected by different methods of Pendimethalin application. Pak. J. Weed Sci. Res. 15(1): 11-17.

Gill, M.I., M. Anwar and D. Mohammad. 1996. Effect of pre-planting irrigation (rouni) and time of pre-emergence application of Stomp $330 \mathrm{E}$ (Pendimethalin) in cotton. Paper read at the $5^{\text {th }}$ Pakistan Weed Science Conference March 1996, NARC, Islamabad.

Gurbachan Singh. 2005. Weed management for sustainable agriculture. National Biennial Conference, ISWS, Ludhiana, Punjab, pp. 1-6.

Hadizadeh, M.H.S., S. Noroozzadeh and H. Rahimion. 2002. Effect of row spacing and weed free period on yield components of cotton (Gossypium hirsutum L.). Applied Entomol. Phytopathal., 69: 171-187.

Hassan, G., N. U. Khan, and Q. Nawaz. 1996. Chemical control of weeds in cotton (G. hirsutum L.) under D.I. Khan Conditions. Paper read at the $1^{\text {st }}$ Crop Protection Conference (CAP) April 21-23, 1996 NWFP Agricultural University Peshawar, Pakistan, Abstracts.pp.41.

Hirematha, K. A. and Sathyanaranyann Rao. 2001. Chemical weed control studies in irrigated hybrid cotton (Gossypium hirsutum L.). Crop Res., 21 (1): 41-45.

Khan, N.U., S. U. Khan, G. Hassan, I. H. Shah and Q. nawaz. 2001. Studies on weed control in cotton (G. hirsutum L.). Online J. Biol. Sci.1 (3): 143-145.

Naqib Ullah Khan, Sana Ullah Khan, Gul Hassan, Inayat Hussain Shah and Qayum Nawaz. 2001. Studies on weed control in cotton (Gossypium hirsutum L.). Online J. Biol. Sci.1 (3): 143-145.

Panwar, R. S., R. S. Malik, S. S. Rathi and R.K. Malik. 2001. Chemical weed control in cotton. Ind. J. Weed sci., 33 (1\&2): 14-17.

Richardson, R. J., H. P. Wilson and T. E. Hines. 2007. Pre-emergence herbicides followed by trifloxysulfuron post-emergence in cotton. Weed Tech., 21 (1): 1-6. 